\title{
The homocysteine controversy
}

\author{
Yvo M. Smulders • Henk J. Blom
}

Received: 11 February 2010 /Revised: 8 May 2010 / Accepted: 2 June 2010 /Published online: 22 June 2010

(C) The Author(s) 2010. This article is published with open access at Springerlink.com

\begin{abstract}
Mild to moderate hyperhomocysteinemia has been identified as a strong predictor of cardiovascular disease, independent from classical atherothrombotic risk factors. In the last decade, a number of large intervention trials using $\mathrm{B}$ vitamins have been performed and have shown no benefit of homocysteine-lowering therapy in high-risk patients. In addition, Mendelian randomization studies failed to convincingly demonstrate that a genetic polymorphism commonly associated with higher homocysteine levels (methylenetetrahydrofolate reductase $677 \mathrm{C}>\mathrm{T}$ ) is a risk factor for cardiovascular disease. Together, these findings have cast doubt on the role of homocysteine in cardiovascular disease pathogenesis, and the homocysteine hypothesis has turned into a homocysteine controversy. In this review, we attempt to find solutions to this controversy. First, we explain that the Mendelian randomization analyses have limitations that preclude final conclusions. Second, several characteristics of intervention trials limit interpretation and generalizability of their results. Finally, the possibility that homocysteine lowering is in itself beneficial but is offset by adverse side effects of $\mathrm{B}$ vitamins on atherosclerosis deserves serious attention. As we explain, such side effects may relate to direct adverse effects of the B-vitamin regimen (in particular, the use of high-dose folic acid) or
\end{abstract}

Communicated by: Brian Fowler

Competing interest: None declared.

Presented at: The International Fulda-Symposium on Homocysteine, Folate and Cobalamin Disorders, Fulda, Germany 11-13 November 2009

Y. M. Smulders $(\bowtie) \cdot$ H. J. Blom

Internal Medicine and Institute for Cardiovascular Research, VU University Medical Center,

ICaR-VU, PO 7057, 1007 MBAmsterdam, The Netherlands

e-mail: y.smulders@vumc.nl to proinflammatory and proproliferative effects of B vitamins on advanced atherosclerotic lesions.

\section{Introduction}

Mild hyperhomocysteinemia has been firmly established as an independent predictor of cardiovascular disease (CVD). A meta-analysis of studies published before 2002 concluded that a $3-\mu \mathrm{mol} / 1$ increase in fasting plasma homocysteine is associated with an $11 \%$ increase in the incidence of ischemic heart disease and a $19 \%$ increase in the incidence of stroke (Homocysteine Studies Collaboration 2002; Hansson 2005). More recent studies confirmed that homocysteine is a strong, independent CVD predictor (de Ruijter et al. 2009). The resulting homocysteine hypothesis states that mild to moderate hyperhomocysteinemia is a causal contributor (i.e., a risk factor) to the occurrence of CVD.

Not all disease predictors are risk factors in the strict definition. A variable may predict disease without being causally related to the disease. The common term for such a predictor is biomarker (alternatively, the term risk marker or risk indicator is used). A biomarker commonly represents an early stage of the disease. Interventions aimed at optimizing a biomarker may or may not be associated with reduced disease incidence depending on whether the treatment has an effect on a causal mechanism that underlies both the appearance of the biomarker as well as the occurrence of disease. Treatment that reduces the biomarker without affecting disease incidence is useless. Recent outcomes of large intervention trials have raised suspicion that mild hyperhomocysteinemia is not a causal risk factor but is, in fact, a predictor of CVD by virtue of a role as biomarker, treatment of which does not reduce clinical disease. A detailed analysis of the intervention 
studies is presented elsewhere in this issue. In essence, the collective evidence shows that treating mild hyperhomocysteinemia (mean baseline homocysteine usually between 10 and $14 \mu \mathrm{mol} / \mathrm{l}$ ) with $\mathrm{B}$ vitamins (folic acid, vitamin $\mathrm{B}_{12}$, and vitamin $\mathrm{B}_{6}$, usually in combination) for a mean period ranging from 2 to 7 years does not reduce the incidence of clinical CVD endpoints.

Additional doubt for a causal role of homocysteine in CVD has been raised in Mendelian randomization analyses. Mendelian randomization is based on the principle that genes are randomly allocated during meiosis, thus creating a "trial of nature" (Davey Smith and Ebrahim 2003). If a gene mutation is responsible for higher plasma homocysteine levels, random allocation of this gene during conception essentially creates a randomized trial of low versus high homocysteine from birth onward. The $\mathrm{C}>\mathrm{T}$ polymorphism in the methylenetetrahydrofolate reductase (MTHFR) enzyme is such an example. Although initial Mendelian randomization analyses supported the homocysteine hypothesis (Hansson 2005; Klerk et al. 2002), recent similar analyses found no consistent effect of the $\mathrm{T}$ allele on CVD (Clarke 2009; Lewis et al. 2005).

Mendelian randomization studies in combination with negative intervention trials have transformed the homocysteine hypothesis into a homocysteine paradox. We argue that abandoning the hypothesis is premature. To make our point, we address limitations of Mendelian randomization and discuss limitations of intervention studies. In addition, we discuss possible dual effects of B-vitamin supplementation on atherosclerosis, with intrinsic beneficial effects of homocysteine lowering being offset by detrimental effects of synthetic folic acid and/or stimulation of components of atherosclerosis by B vitamins.

\section{Limitations of Mendelian randomization}

The principle of Mendelian randomization is illustrated in Fig. 1 (Davey Smith and Ebrahim 2003). Although often considered to provide the most objective type of epidemiological evidence, the validity of a Mendelian randomization analysis is critically dependent on a number of assumptions
(Sheehan et al. 2008), which we discuss below in the context of the homocysteine hypothesis.

- The gene variant should have no relation with any of the confounders.

Whether this assumption is met in the example of MTHFR $677 \mathrm{C}>\mathrm{T}$ and the homocysteine hypothesis is unknown. Certainly, full understanding of the factors that may confound the association between homocysteine and CVD is essential. Given our limited ability to explain population variance of plasma homocysteine levels, we cannot safely exclude the possibility that the MTHFR $677 \mathrm{C}>\mathrm{T}$ polymorphism is not related to any confounder.

- The gene variant has a direct, consistent, and quantifiable association with the risk factor.

This assumption is only partly met in the example of MTHFR $677 \mathrm{C}>\mathrm{T}$ and plasma homocysteine levels. For example, the association is modified by systemic folate status, measurements of which (e.g., serum folate) notably have limited reliability.

- The gene variant has no direct effect on the disease, or no effect other than that mediated via the risk factor of interest.

Whether this assumption is valid for homocysteine is uncertain. The MTHFR enzyme has a key role in what is referred to as the metabolic switch in folate metabolism. The $677 \mathrm{~T}$ allele is probably associated with an increased flux of folate through the DNA-RNA biosynthesis cycle. This is reflected, for example, by increased concentrations of non-methyl-reduced folates in erythrocytes of MTHFR 677 TT individuals (Smulders et al. 2007). This preferential flux of folate through the DNA-RNA biosynthesis cycle has been suggested to play a role in the association between the MTHFR $677 \mathrm{C}>\mathrm{T}$ polymorphism and various other disease, in particular, cancer (Kim 2009). Whether altered folate partitioning at the level of MTHFR also directly (i.e., independently of homocysteine) affects CVD development is unknown but cannot be excluded.

- The risk factor must itself be the cause of disease, not an indirect reflection of a related causal factor.

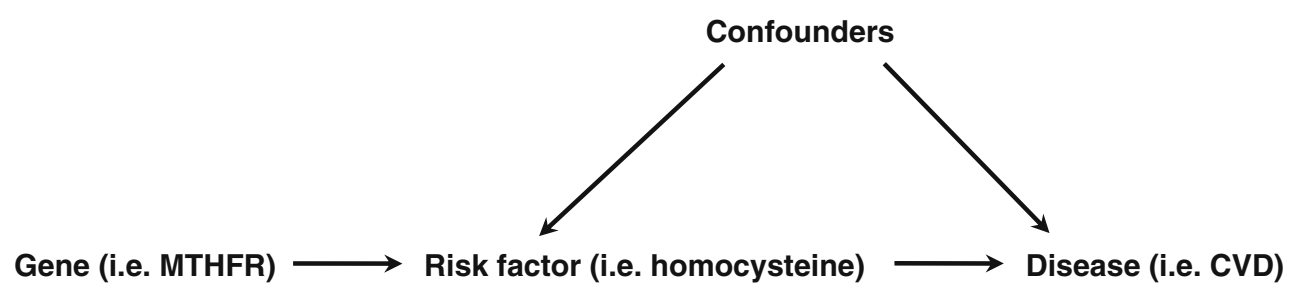

Fig. 1 Principle of Mendelian randomization as applied to hyperhomocysteinemia. This is the ideal model of Mendelian randomization. Essential for the validity of the model is the absence of arrows between the gene and (known or unknown) confounders, as well as the absence of an arrow (i.e., a direct association not dependent on the risk factor) between the gene and the disease. MTHFR methylenetetrahydrofolate reductase, $C V D$ cardiovascular disease 
The importance of this final assumption cannot be understated. Ever since plasma homocysteine was identified as a risk factor for CVD, there has been a lack of knowledge as to whether plasma homocysteine itself is detrimental. Alternatively, raised plasma homocysteine may represent changes in intracellular 1-carbon metabolism that do not respond similarly to B-vitamin treatment, as does plasma homocysteine. How intracellular 1-carbon metabolites relate to plasma homocysteine is, however, unclear. Limited data suggest that, for example, plasma homocysteine relates to intracellular concentrations of the transmethylation inhibitor S-adenosylhomocysteine (Ado-Hcy) (Yi et al. 2000), but whether B vitamins decrease intracellular Ado-Hcy levels is unknown. Clearly, if homocysteine indirectly (and perhaps even weakly) represents some epiphenomenon of an intracellular disturbance in 1-carbon metabolism that causes CVD, the Mendelian randomization study for the MTHFR $677 \mathrm{C}>\mathrm{T}$ genotype loses significance.

A few additional limitations of Mendelian randomization should be mentioned. These limitations include linkage disequilibrium of the studied gene with other genes, genetic heterogeneity, pleiotropy, and population stratification. Finally, an important interpretative limitation is a phenomenon referred to as canalization, which describes the ability of an organism to developmental compensation/adaptation for a disruptive genetic condition.

In conclusion, many, if not all, of the assumptions critical to the validity of Mendelian randomization experiments are either absent or subject to doubt, and Mendelian randomization has intrinsic limitations. The Mendelian randomization study outcomes, therefore, are not suitable to reject the homocysteine hypothesis.

\section{Power of the intervention studies}

The statistical power of an epidemiological study is defined as the probability of identifying a truly existing outcome, commonly a difference between (intervention) groups. Usually, statistical power is interpreted solely in the context of study size. This approach to statistical power carries with it a few implicit assumptions, which impact on the actual ability to observe anticipated effects-for example, of homocysteine-lowering treatment.

Study duration

The estimated effect size of the intervention studies is commonly based on observational epidemiology. Observational findings, however, almost always reflect exposure to the treatment target (e.g., homocysteine) for a longer period of time than the scheduled period of intervention and follow-up. In other words, whereas the observational data relating plasma homocysteine levels to CVD incidence are based on exposure to hyperhomocysteinemia for a duration that may easily exceed 10 years, most intervention trials have a follow-up of $<5$ years.

In the field of CVD, a tradition has emerged to anticipate relatively rapid effects of risk-reducing interventions. Cholesterol lowering by statins, for example, has generated positive results after follow-up durations of $\leq 2$ years. Trials of antihypertensive therapy have shown even more rapid effects. The question now is whether it is reasonable to expect a similarly rapid effect on CVD from any other intervention. The answer is no.

The development of an atherosclerotic plaque is a slow process, commonly taking 30-40 years from initial development to a clinical event. If homocysteine were a true causal contributor to this process, the time span required to show a beneficial intervention effect of lowering homocysteine could intuitively take much longer than the relatively few years of follow-up characterizing the B-vitamin intervention trials. Again, the statin and antihypertensive trials may have put us on a wrong track of expectation. The speed of onset of favorable effects of statins surprised researchers in the field and remains incompletely understood. Conversely, the rapid onset of a favorable effect of antihypertensive therapy was to be expected in the first place, because the mechanical unloading of the heart and vessel wall has immediate antiischemic and plaque-stabilizing effects. There are examples of CVD risk reducing interventions that indeed take much longer than a few years to turn out positive. The best example is glucose lowering. After disappointing results of intensive glucose control on cardiovascular endpoints, extended followup of studies in both type 1 and type 2 diabetes mellitus now suggests that such effects may not become apparent until 10-15 years of follow-up (The DCCT/EDIC Research Group 2005; Holman et al. 2008).

Although it is plausible that the homocysteine-lowering trials have been underpowered simply because the duration of follow-up has been too short, this possibility is rarely even considered. A meta-analysis of the effect of B vitamins on stroke, however, did look at the difference in outcome between studies with $<36$ months and $>36$ months of follow-up. The result of this analysis was a full absence of any effect in the short-term studies but a statistically significant $29 \%$ reduction in the studies with at least 36 months of follow-up (Wang et al. 2007).

Severity of hyperhomocysteinemia

Although the association between plasma homocysteine concentrations and CVD appears linear in epidemiological 
studies, the possibility remains that only treatment of relatively severe degrees of hyperhomocysteinemia generates benefit. The baseline level of homocysteine in the large intervention studies was usually between 10 and $14 \mu \mathrm{mol} / \mathrm{l}$, which is hardly above age-adjusted average population values. Patients with higher levels of hyperhomocysteinemia (e.g., $>20 \mu \mathrm{mol} / \mathrm{l}$ ) were underrepresented in the studies, precluding definite conclusions for this category of patients.

\section{Contrast between intervention groups}

The power of any intervention study is dependent no so much on the effect size of the active treatment, but rather on the contrast generated between the intervention groups. In this respect, several intervention trials have been compromised by B-vitamin food fortification implemented just prior to or during the intervention period. In addition, the use of multivitamins is so widespread that up to $20 \%$ of patients included in the large B-vitamin trials were actually using multivitamins at the time of randomization.

The impact of contrast between the intervention groups has not been sufficiently addressed. In the aforementioned meta-analysis of stroke prevention by B vitamins, however, there was a significant impact of both grain fortification and the degree of homocysteine lowering on the probability of finding a protective effect of $\mathrm{B}$ vitamins on stroke incidence. Intervention effects were not observed in fortified study populations or if the degree of homocysteine lowering was $<20 \%$ compared with baseline (Wang et al. 2007).

In conclusion, although the cumulative intervention studies contain a large number of patients, the actual power to detect an intervention effect may be compromised by a duration of follow-up that is simply too short in relation to the time frame of the development of atherosclerotic disease. In addition, conclusions from intervention studies may not apply to patients with moderate hyperhomocysteinemia-for example, exceeding $20 \mu \mathrm{mol} / \mathrm{l}$ - or to patients not exposed to multivitamins or food fortification with $B$ vitamins.

\section{Dual effects of B vitamins on atherosclerosis}

The interpretation of identical event-free survival rates in clinical trials is intuitively interpreted as being compatible with "no effect" of the active treatment compared with placebo. The more correct phrasing would be, however, that "no net effect" is observed. By this, we mean that the apparent absence of an effect may in fact be the sum of favorable and unfavorable effects, which balance out in the clinical event rate.
It is conceivable that B-vitamin treatment has such dual effects on atherosclerosis. The favorable effect would be reduction of homocysteine levels. The unfavorable effects may relate to several aspects of B-vitamin treatment. We briefly discuss two such aspects: potential adverse effects of unmetabolized folic acid, and stimulation of inflammation (and possibly proliferation) in existing atherosclerotic lesions.

Potential adverse effects of folic acid

Folic acid is a synthetic, oxidized provitamin used for food supplementation and pharmacological formulations mainly because it is cheap and stable during manufacturing, storage, and food processing. Folic acid, however, does not occur in normal human physiology and has no known biological function of its own. It requires reduction to tetrahydrofolate and subsequently needs 1-carbon substitution to commence its task as 1-carbon donor for methylation and DNA-RNA synthesis. During passage of small amounts of folic acid through the gut cells and liver, reduction and 1-carbon substitution is complete, and 5 -methyltetrahydrofolate is by far the most predominant form entering the systemic circulation. When the dose of folic acid exceeds $200 \mu \mathrm{g}$, however, unmetabolized folic acid appears in the systemic circulation (Kelly et al. 1997). The potential adverse effects of circulating folic acid have been understudied. It is possible, however, that folic acid has adverse effects on normal transmembrane folate transport and/or has direct detrimental intracellular effects.

Folate transport across epithelia and into systemic tissues predominantly occurs via the reduced folate carrier, the folate receptor family, and the recently discovered protoncoupled folate transporter (Zhao et al. 2009). The role of the proton-coupled folate transporter is unclear in tissues other than the gut (Zhao et al. 2009). The reduced folate carrier has almost exclusive affinity and transport capacity for reduced folates. The folate receptors, however, can bind different isoforms of folate. The potential problem is that folic acid has an affinity for the folate receptor that is many times stronger than that of reduced folates. This high affinity may result in limited or delayed detachment of folic acid from the receptor after cellular internalization of the receptor-folate complex, which would essentially render folate-receptor-mediated transport ineffective. This may, at least theoretically, cause relative folate deprivation in cells that are dependent on the receptor for folate supply. Whether this problem occurs in reality is speculative. No study, however, has addressed this issue. In addition, knowledge of transmembrane folate transport mechanisms in cells that are important for atherosclerosis, such as endothelial cells, monocytes-macrophages, vascular smooth muscle cells, etc., is incomplete, which adds to the 
concern. We are evaluating whether folic acid may adversely affect transmembrane transport of 5-methyltetrahydrofolate.

Another issue is whether folic acid could have direct adverse effects. Again, the literature has not thoroughly addressed this possibility. A single report, however, suggested that unmetabolized folic acid may adversely affect natural-killer-cell function (Troen et al. 2006). On a molecular level, folic acid may impair enzymes for which reduced folates are natural substrates (Ross et al. 1984). Whether such mechanisms are relevant for clinical diseases is unknown. However, indirect support is emerging from epidemiological studies, such as a study suggesting that folic acid supplements increase prostate cancer risk, whereas folate status resulting from natural folate intake is inversely related to this risk (Figueiredo et al. 2009).

\section{Adverse effects of folate on atherosclerosis}

A final source of concern is that the beneficial effect of homocysteine lowering is offset by folate supplementation having detrimental effects on inflammation and proliferation in atherosclerotic lesions. Atherosclerosis is not a process of passive cholesterol accumulation in the arterial wall but, rather, a disease in which many metabolically active and proliferating cells play a role. Macrophages, for example, are important in the development of atherosclerosis by incorporating and accumulating oxidized low-density-lipoprotein cholesterol particles. Also, activated T cells play a role in atherosclerosis (Hansson 2005). Examples of proliferative processes essential in atherosclerosis include neoangiogenesis of microvessels and smooth muscle cell proliferation (Hansson 2005; Sluimer and Daemen 2009). Given the role of folate in transmethylation reactions and DNA/RNA synthesis, B-vitamin cofactors of 1-carbon metabolic cycles should be considered crucial fuel sources of all metabolically active and proliferating cells, including those responsible for inflammation and proliferation in atherosclerosis.

This hypothesis may implicate that the effects of homocysteine lowering by B vitamins may be dependent upon disease stage. In true primary prevention (i.e., absence of atherosclerotic lesions in any more or less advanced stage of development), the lower homocysteine might still have beneficial effects. However, in patients with significant atherosclerotic lesions already being present (i.e., the elderly) the B vitamins may have detrimental effects, neutralizing the benefit of homocysteine lowering. Whether the hypothesis of disease-stage-dependent effects of B vitamins holds true, and $B$ vitamins indeed have the potential to enhance inflammation and proliferation in atherosclerotic lesions, has never been specifically studied. There is, however, considerable indirect support for this hypothesis from human and other animal cell studies, which we discuss briefly below.
If high B-vitamin status protects against initial atherosclerotic lesion development but enhances the disease process in later stages, this could be reflected in epidemiological findings. Indeed, population-based studies have suggested that whereas high folate status may protect young people from CVD, elderly people (in whom advanced atherosclerosis is commonly present) actually benefit from relative folate deprivation (Giles et al. 1998). In addition, one might expect to see particular beneficial effects of antifolate treatment on atherosclerosis-related clinical events. Accordingly, the antifolate methotrexate appears to have specific anti-CVD properties in rheumatoid arthritis patients, its benefits exceeding that of other anti-inflammatory agents that are without specific antifolate properties (van Halm et al. 2006). Whether methotrexate really can delay atherothrombotic events is being evaluated in a large, randomized clinical trial (Ridker 2009). It is at least remarkable that folate supplementation trials are being performed by some, and antifolate treatment by others, without anyone realizing that the hypotheses underlying these trials are, at least partly, mirror images.

Several animal studies have confirmed that B vitamins indeed delay initial development of atherosclerosis (Dayal and Lentz 2008; Carnicer et al. 2007), but putative detrimental effects of folate on advanced lesions have never been specifically addressed (Dayal and Lentz 2008). We are undertaking such studies. As for the putative detrimental effects of $\mathrm{B}$ vitamins on inflammatory and proliferating cells, folate dependency has been observed for activated macrophages (Xia et al. 2009; Antohe et al. 2005). Folate has been shown to enter and stimulate activated macrophages (Xia et al. 2009), thereby skewing them to a more proatherogenic phenotype. In addition, the fact that cells do not proliferate in folate-deprived media is a well-known phenomenon from in vitro studies. Again, however, no previous study has specifically addressed the possibility of B-vitamin-mediated enhancement of proliferative processes taking place in atherosclerotic lesions.

\section{Conclusions and implications}

Recently, the outcome of Mendelian randomization studies and large intervention trials of homocysteine lowering have cast doubt on the role of $\mathrm{B}$ vitamins and homocysteine in CVD. In our view, what the intervention trials have indeed convincingly shown is that routine administration of highdose multi-B vitamins to patients with (a high risk of) CVD has no net benefit and can thus not be recommended. However, abandoning the field of research into the associations between $\mathrm{B}$ vitamins, homocysteine, and CVD would be unwise. As outlined, the Mendelian randomization experiments have limitations that preclude final conclusions. In 
addition, some characteristics of the intervention trials limit the interpretation of their results. Finally, the possibility that homocysteine lowering is beneficial but is offset by adverse side effects of B-vitamin supplementation on atherosclerosis deserves serious attention. As explained, these side effects may relate to direct adverse effects of the B-vitamin regimen (in particular, the use of high-dose folic acid), or to proinflammatory and proproliferative effects of B vitamins on advanced atherosclerotic lesions.

There are several implications to what we have discussed. First, we need to increase our understanding of how homocysteine damages the vascular system and what ways of preventing such damage, other than high-dose B vitamins, are possible. Second, we must study the potential adverse effects of the various forms of (high-dose) B vitamins in cell and animal experiments. Third, we should use epidemiological data to explore the hypothesis that a high B-vitamin status may indeed prevent the initial development of atherosclerosis, particularly in young people. Intervention trials in such people will be very hard to perform logistically, especially with hard clinical endpoints. Finally, we must continue to perform intervention trials addressing questions that have remained unanswered in the trials performed so far. In particular, these remaining questions relate to the type of intervention (e.g., natural folate versus folic acid), the duration of follow-up (e.g., long-term studies or, at least, extended follow-up of the existing studies), and the target population (e.g., separate studies in patients with more severe degree of hyperhomocysteinemia). Abandoning the homocysteine hypothesis now might well turn out to be a historical mistake.

Awaiting the results of studies to come, what should we do with our high-risk patient? Clearly, an evidencebased recommendation is impossible. The evidence collected so far allows for just a single conclusion: high-dose B-vitamin therapy in all patients at high risk of CVD does not reduce events after 5 years. In view of what we have discussed, the following policy may be considered:

- Do not screen for hyperhomocysteinemia routinely in high-risk patients, but only if:

- An inborn error of 1-carbon metabolism is suspected based on the clinical phenotype

- CVD occurs prematurely (i.e., <50 years, cutoff depending on conventional risk-factor profile) in the patient or his/her family

- B-vitamin depletion is suspected based on history or comorbidity

- Do not treat mild hyperhomocysteinemia routinely, but only if fasting levels exceed a certain threshold of, for example, $20-25 \mu \mathrm{mol} / 1$
- Upon decision to treat, do not use high-dose B-vitamin preparations routinely, but start with a multivitamin containing moderate amounts of folate, $\mathrm{B}_{12}$, and $\mathrm{B}_{6}$.

- Switch to high-dose B vitamins only if homocysteine remains clearly elevated

- Keep an eye on the literature

Open Access This article is distributed under the terms of the Creative Commons Attribution Noncommercial License which permits any noncommercial use, distribution, and reproduction in any medium, provided the original author(s) and source are credited.

\section{References}

Homocysteine Studies Collaboration (2002) Homocysteine and risk of ischemic heart disease and stroke: a meta-analysis. JAMA 288:2015-2022

The Diabetes Control and Complications Trial/Epidemiology of Diabetes Interventions and Complications (DCCT/EDIC) Study Research Group (2005) Intensive diabetes treatment and cardiovascular disease in patients with type 1 diabetes. N Engl J Med 353:2643-2653

Antohe F, Radulescu L, Puchianu E, Kennedy MD, Low PS, Simionescu M (2005) Increased uptake of folate conjugates by activated macrophages in experimental hyperlipemia. Cell Tissue Res 320:277-285

Carnicer R, Navarro A, Arbonqs-Mainar JM, AcÆn S, Guzmbn MA, Surra JC et al (2007) Folic acid supplementation delays atherosclerotic lesion development in apoE-deficient mice. Life Sci 80:638-643

Clarke R (2009) Evidence against a causal association of homocysteine and coronary heart disease: a mendelian randomisation study. Data presented at the 7th international conference on homocysteine metabolism, Prague, Chech Republik

Davey Smith G, Ebrahim S (2003) 'Mendelian randomization': can genetic epidemiology contribute to understanding environmental determinants of disease? Int J Epidemiol 32:1-22

Dayal S, Lentz SR (2008) Murine models of hyperhomocysteinemia and their vascular phenotypes. Arterioscler Thromb Vasc Biol 28:1596-1605

de Ruijter W, Westendorp RG, Assendelft WJ, den Elzen WP, de Craen AJ, le Cessie S et al (2009) Use of framingham risk score and new biomarkers to predict cardiovascular mortality in older people: population based observational cohort study. BMJ 338:a3083

Figueiredo JC, Grau MV, Haile RW, Sandler RS, Summers RW, Bresalier RS et al (2009) Folic acid and risk of prostate cancer: results from a randomized clinical trial. J Natl Cancer Inst 101:432-435

Giles WH, Kittner SJ, Croft JB, Anda RF, Casper ML, Ford ES (1998) Serum folate and risk for coronary heart disease: results from a cohort of US adults. Ann Epidemiol 8:490-496

Hansson GK (2005) Inflammation, atherosclerosis, and coronary artery disease. N Engl J Med 352:1685-1695

Holman RR, Paul SK, Bethel MA, Matthews DR, Neil HA (2008) 10year follow-up of intensive glucose control in type 2 diabetes. $\mathrm{N}$ Engl J Med 359:1577-1589

Kelly P, McPartlin J, Goggins M, Weir DG, Scott JM (1997) Unmetabolized folic acid in serum: acute studies in subjects consuming fortified food and supplements. Am J Clin Nutr 65:1790-1795

Kim YI (2009) Role of the MTHFR polymorphisms in cancer risk modification and treatment. Future Oncol 5:523-542 
Klerk M, Verhoef P, Clarke R, Blom HJ, Kok FJ, Schouten EG et al (2002) MTHFR 677C->T polymorphism and risk of coronary heart disease: a meta-analysis. JAMA 288:2023-2031

Lewis SJ, Ebrahim S, Davey SG (2005) Meta-analysis of MTHFR $677 \mathrm{C}->\mathrm{T}$ polymorphism and coronary heart disease: does totality of evidence support causal role for homocysteine and preventive potential of folate? BMJ 331:1053

Ridker PM (2009) Testing the inflammatory hypothesis of atherothrombosis: scientific rationale for the cardiovascular inflammation reduction trial (CIRT). J Thromb Haemost 7 (Suppl 1):332-339

Ross J, Green J, Baugh CM, MacKenzie RE, Matthews RG (1984) Studies on the polyglutamate specificity of methylenetetrahydrofolate dehydrogenase from pig liver. Biochemistry 23:1796-1801

Sheehan NA, Didelez V, Burton PR, Tobin MD (2008) Mendelian randomisation and causal inference in observational epidemiology. PLoS Med 5:e177

Sluimer JC, Daemen MJ (2009) Novel concepts in atherogenesis: angiogenesis and hypoxia in atherosclerosis. J Pathol 218:7-29

Smulders YM, Smith DEC, Kok RM, Teerlink T, Gellekink HJ, Vaes WHJ et al (2007) Red blood cell folate vitamer distribution in healthy subjects is determined by the methylenetetrahydrofolate reductase C677T polymorphism and by total folate status. J Nutr Biochem 18:693-699
Troen AM, Mitchell B, Sorensen B, Wener MH, Johnston A, Wood B et al (2006) Unmetabolized folic acid in plasma is associated with reduced natural killer cell cytotoxicity among postmenopausal women. J Nutr 136:189-194

van Halm VP, Nurmohamed MT, Twisk JW, Dijkmans BA, Voskuyl AE (2006) Disease-modifying antirheumatic drugs are associated with a reduced risk for cardiovascular disease in patients with rheumatoid arthritis: a case control study. Arthritis Res Ther 8:R151

Wang X, Qin X, Demirtas H, Li J, Mao G, Huo Y et al (2007) Efficacy of folic acid supplementation in stroke prevention: a metaanalysis. Lancet 369:1876-1882

Xia W, Hilgenbrink AR, Matteson EL, Lockwood MB, Cheng JX, Low PS (2009) A functional folate receptor is induced during macrophage activation and can be used to target drugs to activated macrophages. Blood 113:438-446

Yi P, Melnyk S, Pogribna M, Pogribny IP, Hine RJ, James SJ (2000) Increase in plasma homocysteine associated with parallel increases in plasma s-adenosylhomocysteine and lymphocyte dna hypomethylation. J Biol Chem 275:29318 29323

Zhao R, Matherly LH, Goldman ID (2009) Membrane transporters and folate homeostasis: intestinal absorption and transport into systemic compartments and tissues. Expert Rev Mol Med 11:e4 